\title{
Management of scalp psoriasis: current perspectives
}

\author{
This article was published in the following Dove Press journal: \\ Psoriasis: Targets and Therapy \\ 29 March 2016 \\ Number of times this article has been viewed
}

\author{
Kim Blakely' \\ Melinda Gooderham² \\ 'Faculty of Medicine, University of \\ Toronto, Toronto, ON, Canada; \\ ${ }^{2}$ Skin Centre for Dermatology, \\ Peterborough, ON, Canada
}

Correspondence: Melinda Gooderham Skin Centre for Dermatology,

775 Monaghan Rd, Peterborough,

ON K9] 5K2, Canada

Tel +l 7057757546

Fax + I 7057753376

Email mgooderham@centrefordermatology. com
Abstract: Psoriasis is a chronic inflammatory condition. The age of onset, chronicity, physical, and psychosocial consequences of the disease cause psoriasis to have a significant impact on patient quality of life. Scalp psoriasis is no different, and effective treatment results in an improvement in quality of life. Successful management of scalp psoriasis includes topical therapies that are acceptable to the patient for mild-to-moderate disease, and systemic therapies for recalcitrant or moderate-to-severe disease. The most effective topical therapies are corticosteroid products, or combination products with calcipotriol and corticosteroid. Newer vehicle options provide more attractive and pleasing products for patients and may improve adherence. The current perspectives for management of scalp psoriasis are discussed including available data for systemic therapy of severe disease.

Keywords: psoriasis, scalp psoriasis, topical therapies, systemic therapies, biologics

\section{Introduction}

Psoriasis is a chronic inflammatory disorder characterized by well-demarcated, erythematous plaques with thick silver-white scale. ${ }^{1}$ Affecting $1 \%-2 \%$ of the North American population, psoriasis is both a physically and psychologically debilitating disease, impacting on quality of life similar to cancer, diabetes, and depression. ${ }^{2}$ Although psoriasis can present at any age, major peaks of incidence occur around 20 and 60 years of age. ${ }^{3}$ Approximately $80 \%$ of patients with psoriasis have mildto-moderate disease, with up to $5 \%$ of their body surface area affected. ${ }^{4}$ Importantly, psoriasis tends to run a chronic course with remissions and exacerbations. Successful management is dependent on a number of factors including patient education, choice of therapeutics, and adherence to treatment course.

Of those affected by psoriasis, up to $80 \%$ will have involvement of the scalp. ${ }^{5}$ Scalp psoriasis may occur in isolation or in conjunction with other forms of psoriasis. Scalp psoriasis is characterized by red, thickened plaques with silver-white scale, either contained within the hairline, or extending onto the forehead, ears, and posterior neck. ${ }^{6}$ Importantly, scalp psoriasis can be a cause of great physical and social distress, with up to $97 \%$ of affected individuals reporting that the condition interferes in their daily life. ${ }^{7}$ In many cases, scalp psoriasis is associated with intense pruritus, and scale is commonly shed as dandruff creating significant social embarrassment for affected individuals. In a recent multinational survey, $43 \%$ of respondents to a telephone survey identified itch as the most bothersome symptom of their psoriasis. ${ }^{8}$ Moreover, scalp psoriasis can also result in alopecia. Although this is commonly reversible with 
appropriate treatment, increasing evidence links scarring alopecia with chronic, relapsing episodes of the disease. ${ }^{9}$

Several objective measures exist to help quantify the severity of scalp psoriasis. For general plaque psoriasis, common tools include the Psoriasis Area and Severity Index (PASI), the Physician Global Assessment (PGA) or Body Surface Area (BSA) scores. ${ }^{10,11}$ The most commonly employed tools for scalp psoriasis are modifications of the PASI: the Psoriasis Scalp Severity Index (PSSI), the Scalpmodified PASI, and the scalp PGA (ScPGA). Unlike the PASI, which evaluates disease severity on four distinct body surface areas, these indexes take into account erythema, induration, and desquamation of disease affecting the scalp only. Other instruments exist but are less commonly employed. ${ }^{11,12}$ There are also patient self-assessment tools that have been developed, including the Dermatology Life Quality Index as well as the Scalpdex, a quality-of-life instrument that addresses symptoms as well as disease impact. ${ }^{12-14}$ Together, these tools can be used not only to assess symptomatology, but also guide treatment choices and evaluate patient response on a variety of indicators.

Despite the vast array of treatment options available for psoriasis, psoriasis of the scalp remains a difficult treatment area. The presence of hair not only impacts the application and penetration of medications to affected areas, but also strongly influences treatment adherence. Patients commonly complain of the greasy effect of medications in this area, and difficulty removing products from their hair. Currently, topical medications are recommended as a first line of treatment in mild-to-moderate psoriasis but can also be used concomitantly with phototherapy, systemic or biological therapies for moderate-to-severe psoriasis. Topical corticosteroids, with or without the addition of the vitamin D analog calcipotriol, are the gold standard for the treatment of scalp psoriasis. Importantly, in recent years, there have been a number of new formulations introduced to the market (eg, foams, shampoos, and sprays) that enhance cosmetic acceptability and adherence. In severe or recalcitrant cases, systemic treatment should be considered.

\section{Treatment of scalp psoriasis Shampoos}

Hair makes the treatment of scalp psoriasis especially challenging. Vehicle formulations play a large role in patient satisfaction and adherence. Shampoos provide patients with an easy, non-messy treatment option, either alone or in combination with other therapies. Few studies have evaluated the actual efficacy of these preparations, reporting shampoos have a modest, at best, effect on treating psoriasis of the scalp..$^{15}$ There are some studies available that support the use of coal tar shampoo, containing 2\%-10\% coal tar solution, in scalp psoriasis; ${ }^{16,17}$ however, no double-blind studies are available to support this practice and many patients do not find tar shampoos cosmetically acceptable. Salicylic acid shampoo, due to its keratolytic effect, has also been used to enhance the penetration of other topical medications including corticosteroids, and has been recommended by The National Psoriasis Foundation as a first-line therapy. ${ }^{5}$ However, a recent Cochrane review of clinical trials on scalp psoriasis found no evidence to support the first-line use of a tar-based shampoo, with or without a keratolytic such as salicylic acid. ${ }^{18}$ Alternatively, clobetasol propionate (CP) shampoo $0.05 \%$ has been proven to be highly effective for scalp psoriasis. This formulation has been found to be both safe and efficacious for the initial treatment phase of disease, as well as maintenance therapy to prevent disease relapse once a patient has achieved remission with other topical or systemic therapies (see Topical corticosteroids). ${ }^{19-21}$

\section{Topical treatments}

\section{Salicylic acid}

Salicylic acid 5\%-10\% has a potent keratolytic effect. Salicylic acid is generally formulated in an ointment or mineral oil and applied for an extended period to reduce the hyperkeratotic scale characteristic of plaque psoriasis. In such cases, removal of scale can help with the penetration of adjunctive medications. ${ }^{5}$ Due to its formulation, patients frequently report difficulty removing the product from their hair; this can be circumvented by applying a clarifying shampoo to the hair prior to entering the shower to aid in removal of the product.

\section{Coal tar}

Coal tar has been used as a treatment for psoriasis for several decades due to its anti-proliferative and anti-inflammatory actions, and its potent anti-pruritic properties. ${ }^{22}$ Although crude coal tar is the most effective tar available for the treatment of psoriasis, the application of crude coal tar to the scalp presents a challenge. As such, coal tar solution or liquor carbonis detergens $5 \%-20 \%$ is frequently recommended, formulated as a lotion, or added to a corticosteroid preparation. Of note, the use of coal tar has largely fallen out of favor to newer topical medications. Tar's malodor, adverse effects (hair staining and drying), and carcinogenicity limit its use, although newer studies challenge these findings. ${ }^{23,24}$ The use of coal tar in cosmetic preparations has been banned 
in Canada and the European Union over concerns regarding carcinogenicity.

\section{Dithranol (anthralin)}

Dithranol, one of the oldest medications used in the treatment of psoriasis, ${ }^{25}$ works by inducing free radical production, thus inhibiting several aspects in the pathogenesis of psoriasis. ${ }^{26,27}$ Dithranol is commonly prescribed as a $0.1 \%-3 \%$ cream, starting at a low concentration and increasing as tolerated. Dithranol is generally indicated in patients with resistant disease, as treatments are generally provided at specialized centers or inpatient departments. This is another treatment that has been replaced by newer, more cosmetically appealing options.

\section{Topical corticosteroids}

Of all the topical treatments available, corticosteroids have shown the greatest efficacy in clinical trials for scalp psoriasis, and are the most commonly prescribed treatment. ${ }^{5,18}$ Given the importance of vehicle formulations in patient adherence, topical corticosteroids are now available as creams, lotions, ointments, oils, gels, foams, solutions, sprays, and shampoos. Several reports suggest that patients prefer newer cosmetically appealing options such as sprays and foams. Although some caution exists against prescribing potent topical corticosteroids for chronic use in scalp psoriasis, the scalp is relatively resistant to atrophy induced by these medications. $^{5}$

In randomized controlled trials, super-potent $\mathrm{CP}$ in various formulations has been shown to be the most efficacious topical treatment for scalp psoriasis. In a recent vehicle-controlled, randomized, double-blind study involving 81 patients with moderate-to-severe scalp psoriasis, $85 \%$ of patients using CP spray were clear or almost clear of their disease after 4 weeks of twice daily application compared with only $13 \%$ in the vehicle control group $(P<0.001) .^{28}$ Another recent study involving 168 patients with moderate scalp psoriasis evaluated the efficacy of once daily CP $0.05 \%$ shampoo. This study demonstrated similar results, with $42 \%$ of patients using CP shampoo found to be clear or almost clear of their disease after 4 weeks of treatment compared with only $2 \%$ in the vehicle control group $(P<0.001) .{ }^{21}$ Continued twice weekly use of the $\mathrm{CP}$ shampoo provided continued benefit to the treatment group, significantly prolonging the time to first relapse, and increasing the total percentage of patients without relapse. ${ }^{21}$ Importantly, both studies stated similar adverse event profiles between the treatment and vehicle arms, with no significant differences in scalp atrophy, telangiectasias, hypothalamic-pituitary-adrenal axis suppression or other adverse events. A recent Cochrane review found the most evidence for the use of CP in the treatment of scalp psoriasis, with four high-quality clinical trials demonstrating a combined 1.9-point change in participants' psoriasis on a six-point global improvement scale (Investigators Global Assessment of Improvement). ${ }^{18}$

In addition to sprays and shampoos, the foam or mousse vehicle has become popular with many patients. CP foam has also been found to be highly efficacious in the treatment of scalp psoriasis. One randomized, double-blind study in 188 patients with moderate-to-severe scalp psoriasis compared the use of CP foam $0.05 \%$ to both CP solution $0.05 \%$ and placebo. Following a 2 -week treatment period, $74 \%$ of patients treated with CP foam were clear or almost clear of their disease, compared with $63 \%$ in the solution treated group. ${ }^{29}$ Another, more recent, open-label trial on 12 patients with scalp psoriasis used CP $0.05 \%$ foam twice daily for 4 weeks. In this study, the PASI score dropped from 5.7 to 1.7 (range: $0.2-4.8$, standard deviation [SD]: 1.1 ) by week 2 , and to 1.1 (range: $0.2-2.2$, SD: 0.6 ) by week $4 .{ }^{30}$ The study is limited by small size and lack of a control group, but demonstrates the significant benefit that can be derived from the use of corticosteroid foams in the treatment of scalp psoriasis.

Although there are fewer trials to support their benefit, other very potent topical corticosteroids (eg, betamethasone dipropionate) and medium to potent topical corticosteroids (eg, betamethasone valerate [BMV]) were reported to have a similar beneficial effect to $\mathrm{CP} .{ }^{18}$ The medium potency 0.12\% BMV foam (Luxiq ${ }^{\circledR}$; Prestium Pharma, Newtown, PA, USA) was shown to be highly efficacious in the treatment of scalp psoriasis, and to be significantly more efficacious than BMV $0.1 \%$ lotion with enhanced penetration. ${ }^{31}$ In a placebocontrolled study including 179 patients with moderate-tosevere scalp psoriasis, $72 \%$ of patients using BMV foam achieved clear or almost clear status, compared to only $47 \%$ using BMV $0.1 \%$ lotion and $21 \%$ of patients using placebo foam or lotion. ${ }^{31} \mathrm{~A}$ cross-over, multi-center study comparing the foam to standard therapies had 210 patients complete the treatment period. Eighty-eight percent of patients using BMV $0.12 \%$ foam had complete or near complete resolution of scaling compared to only $66 \%$ of patients using standard therapies of corticosteroid or calcipotriol $(P<0.001) .{ }^{32}$ Patients also reported that the foam was a more convenient formulation to use as opposed to standard therapies. ${ }^{32}$ Another trial on 79 patients showed that once daily application of BMV $0.12 \%$ foam was as effective as twice daily application for 
4 weeks. ${ }^{33}$ This is important given that once daily efficacious treatment has a much higher adherence rate as compared to twice daily treatments. ${ }^{34}$

\section{Vitamin $D$ derivatives}

Vitamin D analogs have been shown to induce dosedependent anti-proliferative and pro-differentiating effects in the epidermal keratinocytes of psoriatic skin. ${ }^{35,36}$ Unlike the corticosteroids, vitamin D analogs do not induce skin atrophy, and so have become a regular player in the roster of topical therapies for psoriasis.

The efficacy and long-term safety of calcipotriol has been evaluated in a number of studies. One randomized, double-blind study of 49 patients evaluated the efficacy of twice daily calcipotriol solution $(50 \mathrm{mcg} / \mathrm{mL})$ over a 4 -week treatment period. The study reported that $60 \%$ of patients treated with calcipotriol solution were clear or demonstrated marked improvement in their symptoms as compared with only $17 \%$ in the placebo group. The total sign score for scalp psoriasis (ie, sum of the scores for redness, induration, and scale) decreased by $49 \%$ in the treatment group compared with $19 \%$ in the placebo group $(P=0.005)$. Patients' selfreports also demonstrated that calcipotriol was superior in reducing scalp flaking and itch. ${ }^{37}$

A more recent open-label, non-controlled study evaluating the effect of twice daily treatment of scalp psoriasis with calcipotriol scalp solution $(50 \mathrm{mcg} / \mathrm{mL})$ found the total sign score for scalp psoriasis reduced from 5.9 to $2.5(P<0.001)$ over a 28 -day treatment period. ${ }^{38}$ No further reduction in disease severity was observed with continued treatment for a total of 52 weeks, but the percentage of patients assessing their scalp psoriasis as moderate-to-severe had decreased from $72 \%$ to $21 \%$. There were no significant changes in mean serum calcium, parathyroid hormone or urinary calcium/creatinine ratio. ${ }^{38}$

Studies directly comparing the effects of vitamin D analog monotherapy with very potent or potent steroid monotherapy consistently demonstrate the superiority of corticosteroids for the treatment of scalp psoriasis. A recent analysis of such trials revealed that corticosteroids provided approximately a $10 \%$ improvement in average scores compared with calcipotriol on a six-point global improvement scale (Investigators Global Assessment of Improvement). ${ }^{18}$ In addition, vitamin D derivatives take longer before an optimal improvement can be seen, approximately 8 weeks, as compared with only 2-3 weeks for corticosteroids. ${ }^{39}$ Given the efficacy of both corticosteroids and vitamin D analogs in the treatment of psoriasis, it is not surprising that combination products have been developed and have quickly become a favored medication in the treatment of scalp psoriasis.

\section{Vitamin D/corticosteroid combination products}

A combination product containing calcipotriol $0.005 \%$ and betamethasone dipropionate $0.05 \%(\mathrm{Ca} / \mathrm{BMD})$ (Daivobet ${ }^{\circledR} /$ Dovobet $^{\circledR}$ gel; Leo Pharma Inc., Copenhagen, Denmark) has been found to be superior to its individual ingredients with a fast onset of action, and with no reports of atrophy, striae, purpura, or significant changes in serum calcium. Head-to-head with calcipotriol, more than twice as many patients $(69 \%$ versus $31 \%)$ had absent or very mild disease after only 8 weeks of use. ${ }^{40}$ Two additional long-term studies evaluating the efficacy and tolerability of $\mathrm{Ca} / \mathrm{BMD}$ gel demonstrated similar results. In these studies, patients with scalp psoriasis evaluated for a 52-week treatment period reported absent-mild disease in $92 \%-100 \%$ of visits with $\mathrm{Ca} / \mathrm{BMD}$ gel. ${ }^{41,42}$ In addition, this product has now been tested in the adolescent population and has been shown to be safe and effective. ${ }^{43,44}$ Due to the formulation of $\mathrm{Ca} / \mathrm{BMD}$ gel, use of clarifying shampoo applied to hair prior to entering the shower has been recommended to aid in removal of the product.

This combination of $\mathrm{Ca} / \mathrm{BMD}$ is also being developed into an aerosol foam product with enhanced penetration. This combination foam product has been proven to be both safe and effective for body psoriasis and currently studies are underway in scalp psoriasis..$^{45,46}$

\section{Intralesional therapies}

Intralesional corticosteroids have been applied in practice, although specific studies evaluating the effects of this treatment regimen on scalp psoriasis are lacking. Anecdotal reports of their use exist, and in 2009, the US National Psoriasis Foundation recommended intralesional corticosteroids as second-line treatment for scalp psoriasis. $^{5}$

\section{Systemic treatments}

Scalp psoriasis is not typically treated with systemic therapy unless it is required for recalcitrant or severe cases. Although evidence is lacking for conventional agents such as acitretin, methotrexate, and cyclosporine, a few clinical trials looking at the response of scalp psoriasis to biologic therapy and newer agents have been reported, either as sub-analyses or prespecified end points. Overall, scalp psoriasis does improve in conjunction with improvement of body psoriasis with all systemic therapies.

\section{Oral systemic therapy \\ Apremilast}

Apremilast, an oral PDE4 inhibitor, has been studied in three Phase 3 studies. Two pivotal trials, ESTEEM 1 and ESTEEM 2, have reported significant improvement in scalp psoriasis. ${ }^{47}$ The studies included patients with 
moderate-to-very severe scalp psoriasis ( $\mathrm{ScPGA} \geq 3$ ) at baseline. The study design included a 16-week placebocontrolled period in which patients were randomized to apremilast $30 \mathrm{mg}$ twice daily or placebo. At 16 weeks, the percentage of patients who achieved an ScPGA score of 0 (clear) or 1 (minimal) was significantly greater in the apremilast group (46.5\% versus $17.5 \%$ in ESTEEM 1; $40.9 \%$ versus $17.2 \%$ in ESTEEM 2; $P<0.0001$ for both). ${ }^{47}$ During the open-label period, all patients received apremilast 30 mg twice daily from week 16 to week 32 . In those patients who received apremilast from baseline, $37.4 \%$ and $32.4 \%$ of patients achieved an ScPGA score of 0 or 1 (ESTEEM 1 and ESTEEM 2, respectively). Of the patients initially randomized to placebo who switched to apremilast at week 16, 43.6\% and $50.7 \%$ achieved an improvement in ScPGA score to 0 or 1 at week 32 (ESTEEM 1 and ESTEEM 2, respectively). By the end of week 52, in all patients who were PASI responders, an ScPGA of 0 or 1 was maintained in $83.3 \%$ and $62.5 \%$ of patients receiving apremilast (ESTEEM 1 and ESTEEM 2, respectively) and in $64.1 \%$ and $53.3 \%$ of those initially started on placebo and receiving apremilast in the open-label period only (ESTEEM 1 and ESTEEM 2, respectively). ${ }^{47}$

\section{TNF inhibition}

\section{Etanercept}

The anti-TNF receptor fusion protein, etanercept, has the most clinical trial data supporting its use in scalp psoriasis. Etanercept was given in an open-label trial of 2,555 patients to compare continuous versus interrupted therapy. ${ }^{48} \mathrm{PGA}$ was the primary end point and ScPGA was reported as a secondary end point. All patients (continuous therapy and interrupted therapy groups) were treated with etanercept $50 \mathrm{mg}$ twice weekly for the first 12 weeks of the study. Subsequently, the continuous therapy cohort continued with $50 \mathrm{mg}$ once weekly for an additional 12 weeks; subjects in the interrupted therapy cohort held therapy (if subject achieved PGA $\leq 2$ ) until they lost response, at which point etanercept was restarted at week 16 or 20 and was continued up until the end of the study at week $24 .{ }^{48}$

After 12 weeks of etanercept $50 \mathrm{mg}$ twice weekly, the continuous and interrupted groups achieved a mean percentage improvement from baseline in Psoriasis Scalp Scores of $57.5 \%$ and $58 \%$, respectively. Up to week 24 , after the continuous therapy group remained on $50 \mathrm{mg}$ once weekly, the mean percentage improvement from baseline scalp score was maintained at $57.6 \%$, however the intermittent therapy group dropped to $42.9 \%$ improvement over baseline. ${ }^{48}$

Also reported is a randomized, placebo-controlled trial assessing the safety and efficacy of etanercept for scalp psoriasis over a 24 -week period. ${ }^{49}$ In this study, Group A received etanercept $50 \mathrm{mg}$ twice weekly for 12 weeks, followed by etanercept $50 \mathrm{mg}$ once weekly for 12 weeks $(n=62)$. Group B received placebo twice weekly for 12 weeks followed by 12 weeks of etanercept $50 \mathrm{mg}$ twice weekly ( $\mathrm{n}=62$ ). The primary end point was percentage change in PSSI at week $12 .{ }^{49}$ The etanercept group (Group A) had a mean PSSI improvement at week 12 of $86.8 \%$, compared to only $20.4 \%$ in the placebo group (Group B) $(P<0.0001)$. By week 24, Group A had a mean PSSI improvement of $90.6 \%$, which was not significantly different from Group B, which had 79.1\% improvement subsequent to initiating etanercept therapy at week 13. Eighty-six percent of patients in Group A achieved a PSSI-75 at week 12, compared with only $11 \%$ in Group B $(P<0.0001)$. However, at week 24 , the PSSI-75 rates were similar at $86 \%$ and $72 \%$ for Group A and B, respectively. There was also improvement noted in scalp surface area involvement, with a mean percentage improvement at week 12 in Group A of $84.6 \%$ versus only $14.9 \%$ in Group B. At week 24, Group A maintained this benefit at $84.8 \%$, while Group B showed improvement at $75.4 \%$. Changes in overall PASI improvement were consistent with the PSSI improvements and time to improvement was similar; treatment also provided a high level of patient satisfaction. ${ }^{49}$

\section{Adalimumab}

Although no formal study with the anti-TNF monoclonal antibody adalimumab in scalp psoriasis has been performed, a sub-analysis of the Phase 3 BELIEVE study was recently published. ${ }^{50}$ Of the patients enrolled, 663/730 (91.3\%) had scalp involvement. The BELIEVE study compared adalimumab with or without $\mathrm{Ca} / \mathrm{BMD}$ ointment (applied to body, not scalp) in a randomized, controlled safety and efficacy trial. Patients with baseline scalp psoriasis had more severe disease and were slower to respond initially compared to patients without, but this was no longer significant by week 8 , at which point the majority of patients had achieved a PASI-75 response and continued to improve by week 16. PSSI responses correlated with PASI responses. The median PSSI score in patients at baseline was 14, at week 8 was 1 , and at week 16 was 0 . By week $8,75.6 \%$ of patients had achieved a PSSI response (PSSI $\leq 4)$ and by week 16 , a median decrease from baseline of $100 \%$ (mean $77.2 \% \pm 96.9 \%)^{50}$

\section{Infliximab}

There are no formal studies of the anti-TNF chimeric monoclonal antibody infliximab in scalp psoriasis. However, one report on the results from different body regions 
of the combined three Phase 3 clinical trials (EXPRESS 1, EXPRESS 2, and SPIRIT) showed that the improvement in head and neck psoriasis ( $\geq 75 \%$ and $\geq 90 \%$ improvement) was significant for infliximab $3 \mathrm{mg} / \mathrm{kg}$ and $5 \mathrm{mg} / \mathrm{kg}$ versus placebo. Improvement was consistent with the overall PASI responses in all regions. ${ }^{51}$

Another sub-analysis of the PSUNRISE trial, a trial evaluating the effect of infliximab in patients who failed etanercept, was done looking at disease activity within four designated body regions (scalp, face, nails, and palms/soles) as assessed by investigators using a $10 \mathrm{~cm}$ visual analog scale at weeks 1,10 , and 26 . Improvement was defined as a minimum of $1.1 \mathrm{~cm}$ reduction from baseline in patients who had clinically active disease in all areas. The regional response rate was highest for the scalp at week 10, compared to other areas examined. ${ }^{52}$

\section{IL-12/23 inhibition}

\section{Ustekinumab}

The IL-12/23 inhibitor ustekinumab also does not have any formal studies investigating its benefit in scalp psoriasis. However, there have been case reports on the benefit of this biologic agent in recalcitrant scalp psoriasis. Di Cesare et al and Papadavid et al each present two cases of recalcitrant scalp psoriasis that cleared with ustekinumab by week 8 of treatment. ${ }^{53,54}$

\section{IL- I7a inhibition Ixekizumab}

A monoclonal antibody against IL-17a, ixekizumab, was analyzed in a post hoc analysis of the Phase 2 trial to determine the effect on scalp psoriasis. ${ }^{55}$ A total of 105/142 (74\%) patients enrolled in the Phase 2 program had scalp involvement with a mean baseline PSSI of 18.7 (SD 14.1). At week 12, the percentage improvement of PSSI from baseline was $87.1 \%$ (25 mg), 94.8\% (75 mg), and 84.8\% (150 mg) compared to $30.5 \%$ (placebo). By week 20, a PSSI of 0 , or clear, was achieved in $58.3 \%, 66.7 \%$, and $86.4 \%$ of patients receiving ixekizumab $(25 \mathrm{mg}, 75 \mathrm{mg}$, and $150 \mathrm{mg}$, respectively) compared to only $10 \%$ in the placebo group. At the end of the open-label period at week 48, all patients were receiving ixekizumab $120 \mathrm{mg}$ every 4 weeks, and $78 \%$ of all patients had a PSSI of $0 .{ }^{55}$

\section{Secukinumab}

Secukinumab, another monoclonal antibody against IL-17a, which was approved for treatment of plaque psoriasis in 2014, currently has a Phase $3 \mathrm{~b}$ trial in progress for patients with scalp psoriasis. Approximately 94 subjects who have a PSSI $\geq 12$, a modified Investigators Global Assessment of $\geq 3$ and $\geq 30 \%$ scalp area affected are included in the study. This 24-week study has a 12-week placebo-controlled period and is comparing secukinumab $300 \mathrm{mg}$ to placebo in a $1: 1$ ratio. ${ }^{56}$ Results of this study are eagerly awaited.

\section{Other physical therapies}

\section{Ultraviolet therapy}

Typical ultraviolet (UV) therapy, including narrowband UVB or Psoralen UVA (PUVA) therapy is not practical for the scalp. Modalities to enhance delivery, such as UVB comb, have been developed and tested. In one trial, 14 subjects were treated with a fiber-optic UVB comb three times weekly for 12 weeks, with an area of scalp reserved as a control site. In this study, the treatment sites showed a mean improvement in the modified PASI score of 3.6, with a difference in mean modified PASI between the treatment and control sites of $3.9(P<0.0001) .{ }^{57}$

\section{Laser}

Laser and light sources to treat scalp psoriasis are a challenge due to the presence of hair. One study used a $308 \mathrm{~nm}$ excimer laser, a UV light source with a blowing device to part the hair for optimal delivery. Of the patients enrolled, all had failed class 1 topical steroids along with many other topical therapies. A total of 13/15 patients completed the study and all acted as their own control with only half of the scalp being treated. ${ }^{58}$ After a mean of 29 treatments, there was a 4-point improvement in modified PASI between control and treated areas $(P<0.0001) .^{58}$

\section{Conclusion}

Psoriasis, including scalp psoriasis, is a chronic, recurrent inflammatory condition that has a profound impact on patient quality of life. Many patients report distress related to physical appearance as well as itching, scaling, and in some cases, alopecia. The mainstay of treatment is topical therapy, but this can be challenging given the location and hair-bearing skin. Adherence is an issue and newer topical formulations, such as foams and sprays, help to improve treatment outcomes by improving adherence. In those patients with moderate-to-severe disease who do not respond to topical treatments, systemic therapy needs to be considered.

\section{Disclosure}

Dr Melinda Gooderham is an investigator, advisory board member, speaker for AbbVie, Amgen, Boehringer Ingelheim, Celgene, Eli Lilly, Galderma, Janssen, Leo Pharma Inc., 
Novartis, and Pfizer. The authors have no other conflicts of interest to disclose.

\section{References}

1. Lowes MA, Suarez-Farinas M, Krueger JG. Immunology of psoriasis. Annu Rev Immunol. 2014;32:227-255.

2. Rapp SR, Feldman SR, Exum ML, Fleischer AB Jr, Reboussin DM. Psoriasis causes as much disability as other major medical diseases. J Am Acad Dermatol. 1999;41(3 Pt 1):401-407.

3. Henseler T, Christophers E. Psoriasis of early and late onset: characterization of two types of psoriasis vulgaris. J Am Acad Dermatol. $1985 ; 13(3): 450-456$.

4. Menter A, Gottlieb A, Feldman SR, et al. Guidelines of care for the management of psoriasis and psoriatic arthritis: Section 1. Overview of psoriasis and guidelines of care for the treatment of psoriasis with biologics. J Am Acad Dermatol. 2008;58(5):826-850.

5. Chan CS, Van Voorhees AS, Lebwohl MG, et al. Treatment of severe scalp psoriasis: from the Medical Board of the National Psoriasis Foundation. J Am Acad Dermatol. 2009;60(6):962-971.

6. Papp K, Berth-Jones J, Kragballe K, Wozel G, de la Brassinne M. Scalp psoriasis: a review of current topical treatment options. J Eur Acad Dermatol Venereol. 2007;21(9):1151-1160.

7. Crowley J. Scalp psoriasis: an overview of the disease and available therapies. J Drugs Dermatol. 2010;9(8):912-918.

8. Lebwohl MG, Bachelez H, Barker J, et al. Patient perspectives in the management of psoriasis: results from the population-based Multinational Assessment of Psoriasis and Psoriatic Arthritis Survey. J Am Acad Dermatol. 2014;70(5):871-881.

9. George SM, Taylor MR, Farrant PB. Psoriatic alopecia. Clin Exp Dermatol. 2015;40(7):717-721.

10. Fredriksson T, Pettersson U. Severe psoriasis-oral therapy with a new retinoid. Dermatologica. 1978;157(4):238-244.

11. Spuls PI, Lecluse LL, Poulsen ML, Bos JD, Stern RS, Nijsten T. How good are clinical severity and outcome measures for psoriasis? quantitative evaluation in a systematic review. J Invest Dermatol. 2010;130(4):933-943.

12. Chalmers RJ. Assessing psoriasis severity and outcomes for clinical trials and routine clinical practice. Dermatol Clin. 2015;33(1):57-71.

13. Finlay AY, Khan GK. Dermatology Life Quality Index (DLQI)-a simple practical measure for routine clinical use. Clin Exp Dermatol. 1994;19(3):210-216.

14. Chen SC, Yeung J, Chren MM. Scalpdex: a quality-of-life instrument for scalp dermatitis. Arch Dermatol. 2002;138(6):803-807.

15. van de Kerkhof PC, Franssen ME. Psoriasis of the scalp. Diagnosis and management. Am J Clin Dermatol. 2001;2(3):159-165.

16. Olansky S. Whole coal tar shampoo: a therapeutic hair repair system. Cutis. 1980;25(1):99-104.

17. Lowe NJ, Breeding JH, Wortzman MS. New coal tar extract and coal tar shampoos. Evaluation by epidermal cell DNA synthesis suppression assay. Arch Dermatol. 1982;118(7):487-489.

18. Mason AR, Mason JM, Cork MJ, Hancock H, Dooley G. Topical treatments for chronic plaque psoriasis of the scalp: a systematic review. Br J Dermatol. 2013;169(3):519-527.

19. Jarratt M, Breneman D, Gottlieb AB, Poulin Y, Liu Y, Foley V. Clobetasol propionate shampoo $0.05 \%$ : a new option to treat patients with moderate to severe scalp psoriasis. J Drugs Dermatol. 2004;3(4): 367-373.

20. Reygagne P, Mrowietz U, Decroix J, et al. Clobetasol propionate shampoo $0.05 \%$ and calcipotriol solution $0.005 \%$ : a randomized comparison of efficacy and safety in subjects with scalp psoriasis. $J$ Dermatolog Treat. 2005;16(1):31-36.

21. Poulin Y, Papp K, Bissonnette R, et al. Clobetasol propionate shampoo $0.05 \%$ is efficacious and safe for long-term control of moderate scalp psoriasis. J Dermatolog Treat. 2010;21(3):185-192.

22. Arnold WP. Tar. In: van de Kerkhof PC, editor. The management of psoriasis. Clin Dermatol. 1997;15:739-744.
23. Wheeler LA, Saperstein MD, Lowe NJ. Mutagenicity of urine from psoriatic patients undergoing treatment with coal tar and ultraviolet light. J Invest Dermatol. 1981;77(2):181-185.

24. Roelofzen JH, Aben KK, Oldenhof UT, et al. No increased risk of cancer after coal tar treatment in patients with psoriasis or eczema. J Invest Dermatol. 2010;130(4):953-961.

25. Ashton RE, Andre P, Lowe NJ, Whitefield M. Anthralin: historical and current perspectives. J Am Acad Dermatol. 1983;9(2):173-192.

26. Fuchs J, Packer L. Investigations of anthralin free radicals in model systems and in skin of hairless mice. J Invest Dermatol. 1989;92(5):677-682.

27. Mahrle G, Bonnekoh B, Wevers A, Hegemann L. Anthralin: how does it act and are there more favourable derivatives? Acta Derm Venereol Suppl (Stockh). 1994;186:83-84.

28. Sofen H, Hudson CP, Cook-Bolden FE, et al. Clobetasol propionate $0.05 \%$ spray for the management of moderate-to-severe plaque psoriasis of the scalp: results from a randomized controlled trial. J Drugs Dermatol. 2011;10(8):885-892.

29. Franz TJ, Parsell DA, Myers JA, Hannigan JF. Clobetasol propionate foam $0.05 \%$ : a novel vehicle with enhanced delivery. Int J Dermatol. 2000;39(7):535-538.

30. Mazzotta A, Esposito M, Carboni I, Schipani C, Chimenti S. Clobetasol propionate foam $0.05 \%$ as a novel topical formulation for plaque-type and scalp psoriasis. J Dermatolog Treat. 2007;18(2):84-87.

31. Franz TJ, Parsell DA, Halualani RM, Hannigan JF, Kalbach JP, Harkonen WS. Betamethasone valerate foam $0.12 \%$ : a novel vehicle with enhanced delivery and efficacy. Int J Dermatol. 1999;38(8):628-632.

32. Andreassi L, Giannetti A, Milani M; Scale Investigators Group. Efficacy of betamethasone valerate mousse in comparison with standard therapies on scalp psoriasis: an open, multicentre, randomized, controlled, crossover study on 241 patients. Br J Dermatol. 2003;148(1):134-138.

33. Feldman SR, Ravis SM, Fleischer AB Jr, et al. Betamethasone valerate in foam vehicle is effective with both daily and twice a day dosing: a single-blind, open-label study in the treatment of scalp psoriasis. J Cutan Med Surg. 2001;5(5):386-389.

34. Zaghloul SS, Goodfield MJ. Objective assessment of compliance with psoriasis treatment. Arch Dermatol. 2004;140(4):408-414.

35. Reichrath J, Muller SM, Kerber A, Baum HP, Bahmer FA. Biologic effects of topical calcipotriol (MC 903) treatment in psoriatic skin. J Am Acad Dermatol. 1997;36(1):19-28.

36. Reichrath J, Perez A, Muller SM, et al. Topical calcitriol (1,25dihydroxyvitamin D3) treatment of psoriasis: an immunohistological evaluation. Acta Derm Venereol. 1997;77(4):268-272.

37. Green C, Ganpule M, Harris D, et al. Comparative effects of calcipotriol (MC903) solution and placebo (vehicle of MC903) in the treatment of psoriasis of the scalp. Br J Dermatol. 1994;130(4):483-487.

38. Barnes L, Altmeyer P, Forstrom L, Stenstrom MH. Long-term treatment of psoriasis with calcipotriol scalp solution and cream. Eur J Dermatol. 2000;10(3):199-204.

39. van der Vleuten CJ, van de Kerkhof PC. Management of scalp psoriasis: guidelines for corticosteroid use in combination treatment. Drugs. 2001;61(11):1593-1598.

40. Kragballe K, Hoffmann V, Tan J, et al. Calcipotriene plus betamethasone dipropionate gel compared to calcipotriene solution in patients with scalp psoriasis. J Am Acad Dermatol. 2008;58(2 Suppl 2):AB131.

41. Luger TA, Cambazard F, Larsen FG, et al. A study of the safety and efficacy of calcipotriol and betamethasone dipropionate scalp formulation in the long-term management of scalp psoriasis. Dermatology. 2008;217(4):321-328.

42. Tyring S, Mendoza N, Appell M, et al. A calcipotriene/betamethasone dipropionate two-compound scalp formulation in the treatment of scalp psoriasis in Hispanic/Latino and Black/African American patients: results of the randomized, 8-week, double-blind phase of a clinical trial. Int J Dermatol. 2010;49(11):1328-1333.

43. Gooderham M, Debarre JM, Keddy-Grant J, Xu Z, Kurvits M, Goodfield M. Safety and efficacy of calcipotriol plus betamethasone dipropionate gel in the treatment of scalp psoriasis in adolescents 12-17 years of age. Br J Dermatol. 2015;171(6):1470-1477. 
44. Eichenfield LF, Ganslandt C, Kurvits M, Schlessinger J. Safety and efficacy of calcipotriene plus betamethasone dipropionate topical suspension in the treatment of extensive scalp psoriasis in adolescents ages 12 to 17 years. Pediatr Dermatol. 2015;32(1):28-35.

45. Taraska V, Tuppal R, Olesen M, Bang Pedersen C, Papp K. A Novel Aerosol Foam Formulation of Calcipotriol and Betamethasone Has No Impact on HPA Axis and Calcium Homeostasis in Patients With Extensive Psoriasis Vulgaris. J Cutan Med Surg. 2016;20(1):44-51.

46. Queille-Roussel C, Olesen M, Villumsen J, Lacour JP. Efficacy of an innovative aerosol foam formulation of fixed combination calcipotriol plus betamethasone dipropionate in patients with psoriasis vulgaris. Clin Drug Investig. 2015;35(4):239-245.

47. Rich P, Gooderham M, Bachelez H, et al. Apremilast, an oral phosphodiesterase 4 inhibitor, in patients with difficult-to-treat nail and scalp psoriasis: Results of 2 phase III randomized, controlled trials (ESTEEM 1 and ESTEEM 2). J Am Acad Dermatol. 2016;74(1):134-142.

48. Moore A, Gordon KB, Kang S, et al. A randomized, open-label trial of continuous versus interrupted etanercept therapy in the treatment of psoriasis. J Am Acad Dermatol. 2007;56(4):598-603.

49. Bagel J, Lynde C, Tyring S, Kricorian G, Shi Y, Klekotka P. Moderate to severe plaque psoriasis with scalp involvement: a randomized, doubleblind, placebo-controlled study of etanercept. J Am Acad Dermatol. 2012;67(1):86-92.

50. Thaci D, Unnebrink K, Sundaram M, Sood S, Yamaguchi Y. Adalimumab for the treatment of moderate to severe psoriasis: subanalysis of effects on scalp and nails in the BELIEVE study. J Eur Acad Dermatol Venereol. 2015;29(2):353-360.
51. Menter A, Reich K, Li S, et al. Consistency of infliximab response in different body regions for treatment of moderate to severe psoriasis: Results from controlled clinical trials. J Am Acad Dermatol. 2008;58(suppl: AB120). Abstract 2607.

52. Kalb RE, Blauvelt A, Sofen HL, et al. Effect of infliximab on health-related quality of life and disease activity by body region in patients with moderate-to-severe psoriasis and inadequate response to etanercept: results from the PSUNRISE trial. J Drugs Dermatol. 2013;12(8):874-880.

53. Di Cesare A, Fargnoli MC, Peris K. Rapid response of scalp psoriasis to ustekinumab. Eur J Dermatol. 2011;21(6):993-994.

54. Papadavid E, Ferra D, Koumaki D, et al. Ustekinumab induces fast response and maintenance of very severe refractory scalp psoriasis: results in two Greek patients from the psoriasis hospital-based clinic. Dermatology. 2014;228(2):107-111.

55. Langley RG, Rich P, Menter A, et al. Improvement of scalp and nail lesions with ixekizumab in a phase 2 trial in patients with chronic plaque psoriasis. J Eur Acad Dermatol Venereol. 2015;29(9):1763-1770.

56. Lebwohl M, Qureshi A, Kianifard F et al. Secukinumab in the treatment of moderate to severe scalp psoriasis: A study to evaluate efficacy and safety. J Am Acad Dermatol. 2015;72(5 suppl 1):AB250.

57. Taneja A, Racette A, Gourgouliatos Z, Taylor CR. Broad-band UVB fiber-optic comb for the treatment of scalp psoriasis: a pilot study. Int J Dermatol. 2004;43(6):462-467.

58. Taylor CR, Racette AL. A 308-nm excimer laser for the treatment of scalp psoriasis. Lasers Surg Med. 2004;34(2):136-140.
Psoriasis: Targets and Therapy

\section{Publish your work in this journal}

Psoriasis: Targets and Therapy is international, peer-reviewed, open access journal focusing on psoriasis, nail psoriasis, psoriatic arthritis and related conditions, identification of therapeutic targets and the optimal use of integrated treatment interventions to achieve improved outcomes and quality of life. The manuscript management system

\section{Dovepress}

is completely online and includes a very quick and fair peer-review system. Visit http://www.dovepress.com/testimonials.php to read real quotes from published authors. 